\title{
MODELLING OF SUBCRITICAL FREE-SURFACE FLOW OVER AN INCLINED BACKWARD-FACING STEP IN A WATER CHANNEL
}

\author{
Jaromír PŘíHODA, Pavel ZUBíK, Jan ŠULC, Milan SEDLÁŘ•
}

\begin{abstract}
The contribution deals with the experimental and numerical modelling of subcritical turbulent flow in an open channel with an inclined backward-facing step. The step with the inclination angle $\alpha=20^{\circ}$ was placed in the water channel of the cross-section 200x200 mm. Experiments were carried out by means of the PIV and LDA measuring techniques. Numerical simulations were executed by means of the commercial software ANSYS CFX 12.0. Numerical results obtained for twoequation models and EARSM turbulence model completed by transport equations for turbulent energy and specific dissipation rate were compared with experimental data. The modelling was concentrated particularly on the development of the flow separation and on the corresponding changes of free surface.
\end{abstract}

\section{INTRODUCTION}

Flow over an inclined backward-facing step in closed channel is an intermediate step from flow in a one-sided diffuser to the flow over a backward-facing step with an abrupt change of the cross-section and with flow separation on the step edge. The flow pattern is relatively complicated in spite of a simple geometry because of it includes an extensive separation region and secondary flows near side walls behind the inclined step.

For flow in open channels with an inclined step, the gravitational forces play in important role and the flow character is quite different for sub- and supercritical flow. The subcritical flow is characterized by a relatively massive separation but only small changes of free surface. Results of the investigation of turbulent flow over an inclined step in the closed channel and in the open channel with sub- and supercritical flow are summarized by Zubík at al. [1]. The presented contribution deals with modelling of subcritical flow characterised by the Froude number $\mathrm{Fr}=0.42$.

\section{EXPERIMENTAL ARRANGEMENT}

The test rig was formed by a straight channel of constant cross-section $0.2 \times 0.2 \mathrm{~m}$ with the length $4.475 \mathrm{~m}$ and with the slope of the bottom $0.23 \mathrm{deg}$ corresponding to the chosen subcritical regime. The open channel was linked to the water tank with a pump driven by a motor equipped with a frequency converter. The pump with the maximum flow rate of $Q=7.2 \mathrm{~m}^{3} / \mathrm{min}$. enabled the maximum speed in the channel of $3 \mathrm{~m} / \mathrm{s}$. The channel was made of transparent plastic material allowing the use of contactless optical measuring techniques. To stabilize the inflow rate, a wire mesh screens and a honeycomb were installed at the entry of the straight inflow part.

\footnotetext{
- Jaromír Př́hoda, Institute of Thermomechanics AS CR, v.v.i., Dolejškova 1402/5, 18200 Praha 8; prihoda@it.cas.cz

Pavel Zubík, Jan Šulc, Institute of Hydraulic Structures, Faculty of Civil Engineering, Brno University of Technology, Veveří 331/95, 60200 Brno; Sulc.J@fce.vutbr.cz, Zubik.P@fce.vutbr.cz Milan Sedláŕ, SIGMA - Research and Development Institute, Jana Sigmunda 79, 78350 Lutín; milan.sedlar@sigma-vvu.cz
} 
Two optical methods were used - the particle image velocimetry for measurements of the velocity field in selected channel sections, and the laser Doppler anemometry for measurements of one and/or two velocity components in selected points of the flow field. The extended uncertainty guess of flow velocity determination at reliability level of $95 \%$ was less than $5 \%$ for LDA and $15 \%$ for PIV techniques.

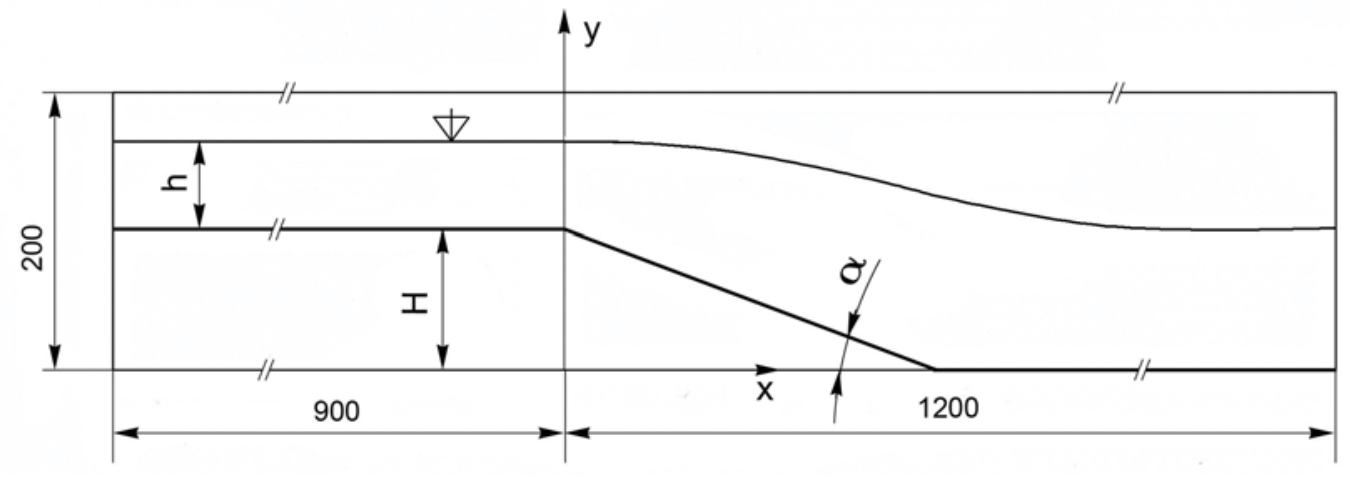

Fig.1 Sketch of the geometrical arrangement

The sketch of the geometrical arrangement of the channel with an inclined backwardfacing step is given in Fig. 1. The distance of the edge of the inclined step from the channel entry was $0.9 \mathrm{~m}$. The height of the inclined step is $H=100 \mathrm{~mm}$. The whole length of the investigated section was $2.1 \mathrm{~m}$. Boundary conditions for the numerical simulation were examined in detail in the cross-sections $x=-0.9$ and $-0.6 \mathrm{~m}$ using the PIV and LDA methods. At the chosen mean bulk velocity, the turbulence level in the stream core was about $2.5 \%$. The PIV measurements were taken at vertical and horizontal planes parallel with the channel axis with the aim to determine the general view of the flow with separation and secondary flows behind the step as well. In the mean vertical plane, profiles of mean and fluctuation longitudinal velocities were determined by the LDA method at selected sections $x=$ const. The measurement of the subcritical free-surface flow was carried out for the mean bulk velocity $U_{m}=0.38 \mathrm{~m} / \mathrm{s}$ and for the initial height of the water level $h=0.082 \mathrm{~m}$, i.e. for the Reynolds number $R e_{b}$ $=U_{m} H / v=44100$ and the Froudé number $\mathrm{Fr}=U_{m} /(g h)^{1 / 2}=0.42$.

\section{Mathematical MOdel}

The numerical simulation of the turbulent flow over the inclined step in an open channel was carried out by means of the commercial software ANSYS CFX version 12.0 solving Reynolds-averaged Navier-Stokes equations including the gravity effect. A second-order scheme was used for calculations. The numerical solution of free-surface flow was carried out by means of the Volume-of-Fluid (VOF) method. This method is based on the monitoring of the volume fraction $\alpha_{i}$ of both fluids in the each computational cell. The free surface is determined as the interface where $\alpha_{\text {water }}=\alpha_{\text {air }}=0.5$ is valid.

The standard $k-\varepsilon$ model according to Launder and Sharma [2], the SST $k-\omega$ model proposed by Menter [3] and the EARSM (Explicit Algebraic Reynolds Stress Model) based on transport equations for turbulent energy $k$ and specific dissipation rate $\omega$ were tested for the subcritical flow. Besides, the SST-SAS (Scale Adaptive Simulation) model proposed by Menter and Egorov [4] was tested from the viewpoint of the possibility of modelling large vortices in the channel flow with separation.

The computational domain corresponding to the experimental arrangement starts in the cross-section $x=-0.6 \mathrm{~mm}$ before the edge of the inclined step and finishes at the distance $x=1.2 \mathrm{~m}$. The computational domain consists of one half of the channel width 
with the symmetric boundary condition in the symmetry plane $(z=0)$. The computational grid in the channel with the inclines step is shown in Fig.2. A structured mono-block type grid refined near walls and near the free-surface was used for calculations. The grid refinement was adapted to the shape of free surface obtained by preliminary calculations. According to the initial height of the free surface, the height of the computation domain $0.2 \mathrm{~m}$ was chosen. The grid consists of $367 \times 169 \times 51$ grid points, i.e. approximately $3.1 \times 10^{6}$ grid points.

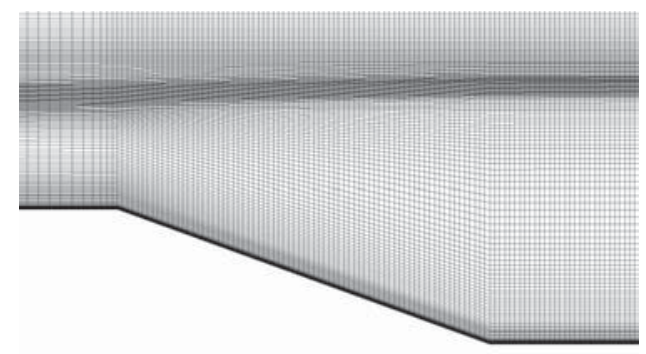

Fig.2 Detail of the computational grid

The distribution of mean velocity, turbulent energy, and dissipation rate was prescribed as inflow boundary conditions according to experimental data. The mean value of static pressure was prescribed as the outflow boundary condition and the open-boundary condition was applied at the upper boundary. The scalable wall functions according Grotjans and Menter [5] were used as wall boundary conditions in calculations based on the $k-\omega$ model while the $k-\varepsilon$ model uses standard wall functions. The non-dimensional distance $y^{+}$of the first grid point from the wall was $y^{+}=1 \div 5$ in the $k-\omega$ model and $y^{+} \approx 10$ in the $k-\varepsilon$ model.

The two-equation $k-\omega$ model in various modifications was used as a basic turbulence model. The $k-\omega$ model is formed by transport equations

$$
\begin{aligned}
& \frac{D k}{D t}=P_{k}-c_{\mu} \omega k+\frac{\partial}{\partial x_{j}}\left[\left(v+\frac{v_{t}}{\sigma_{k}}\right) \frac{\partial k}{\partial x_{j}}\right] \\
& \frac{D \omega}{D t}=\alpha \frac{\omega}{k} P_{k}-\beta \omega^{2}+\frac{\partial}{\partial x_{j}}\left[\left(v+\frac{v_{t}}{\sigma_{\omega}}\right) \frac{\partial \omega}{\partial x_{j}}\right]+\left(1-F_{1}\right) \frac{2}{\sigma_{\omega 2}} \frac{1}{\omega} \frac{\partial k}{\partial x_{j}} \frac{\partial \omega}{\partial x_{j}}+Q_{S A S}
\end{aligned}
$$

with turbulent viscosity given by the relation

$v_{t}=\frac{a_{1} k}{\min \left(a_{1} \omega ; F_{2} S\right)}$

where $F_{1}$ and $F_{2}$ are blending function for the switching from the $k-\varepsilon$ mode to the $k-\omega$ mode and $S$ is a scalar invariant of the strain rate tensor. The function $F_{2}$ is equal to zero everywhere for the BSL model. The additional source term $\mathrm{Q}_{S A S}$ included in the SAS model contains two length scales. Besides to the modelled length scale $L=k^{1 / 2} /\left(c_{\mu}{ }^{1 / 4} \omega\right)$, it is the Karman length scale allowing the adjustment of the SAS model to resolved turbulent structures in the URANS mode with the LES-like behaviour in unsteady computations. More details including model constants and blending functions can be found in Egorov and Menter [6].

The EARSM model represents a simplification of the Reynolds stress models and it is based on a nonlinear relation between the Reynolds stresses and the mean strain rate and vorticity tensors. The used version corresponds to the models proposed by Wallin and Johansson [7] and Hellsten [8]. The constitutive relation for the turbulent viscosity is replaced by the introduction of the anisotropy tensor $a_{i j}$ by the relation 


$$
a_{i j}=\frac{\overline{u_{i} u_{j}}}{k}-\frac{2}{3} \delta_{i j}
$$

The anisotropy tensor $a_{i j}$ is given by the equation

$$
\begin{aligned}
& a_{i j}=\beta_{1} S_{i j}+\beta_{3}\left(\Omega_{i k} \Omega_{k j}-\frac{1}{3} \delta_{i j} I I_{\Omega}\right)+\beta_{4}\left(S_{i k} \Omega_{k j}-\Omega_{i k} S_{k j}\right) \\
& +\beta_{6}\left(S_{i k} \Omega_{k l} \Omega_{l j}+\Omega_{i k} \Omega_{k l} S_{l j}-\frac{2}{3} \delta_{i j} I V\right)+\beta_{9}\left(\Omega_{i k} S_{k l} \Omega_{l m} \Omega_{m j}-\Omega_{i k} \Omega_{k l} S_{l m} \Omega_{m j}\right)
\end{aligned}
$$

where $S_{i j}$ and $\Omega_{i j}$ are non-dimensional strain rate and vorticity tensors, and $I I_{S}, I I_{\Omega}$ and $I V$ are their invariants. The coefficients $\beta_{i}$ are functions of the ratio $P_{k} / \varepsilon$ and invariants of the strain-rate and vorticity tensors. The model is closed by the transport equations for turbulent scales.

The "non-homogeneous" model where the governing equations for the both fluids are solved separately was chosen for the calculation. Calculations by the standard twoequation $k-\varepsilon$ and $k-\omega$ models and by the EARSM models were accomplished as steady, while the calculations by the SAS model were made as unsteady with 3D unsteady velocity fluctuations superposed on the mean velocity profile as the inlet boundary condition.
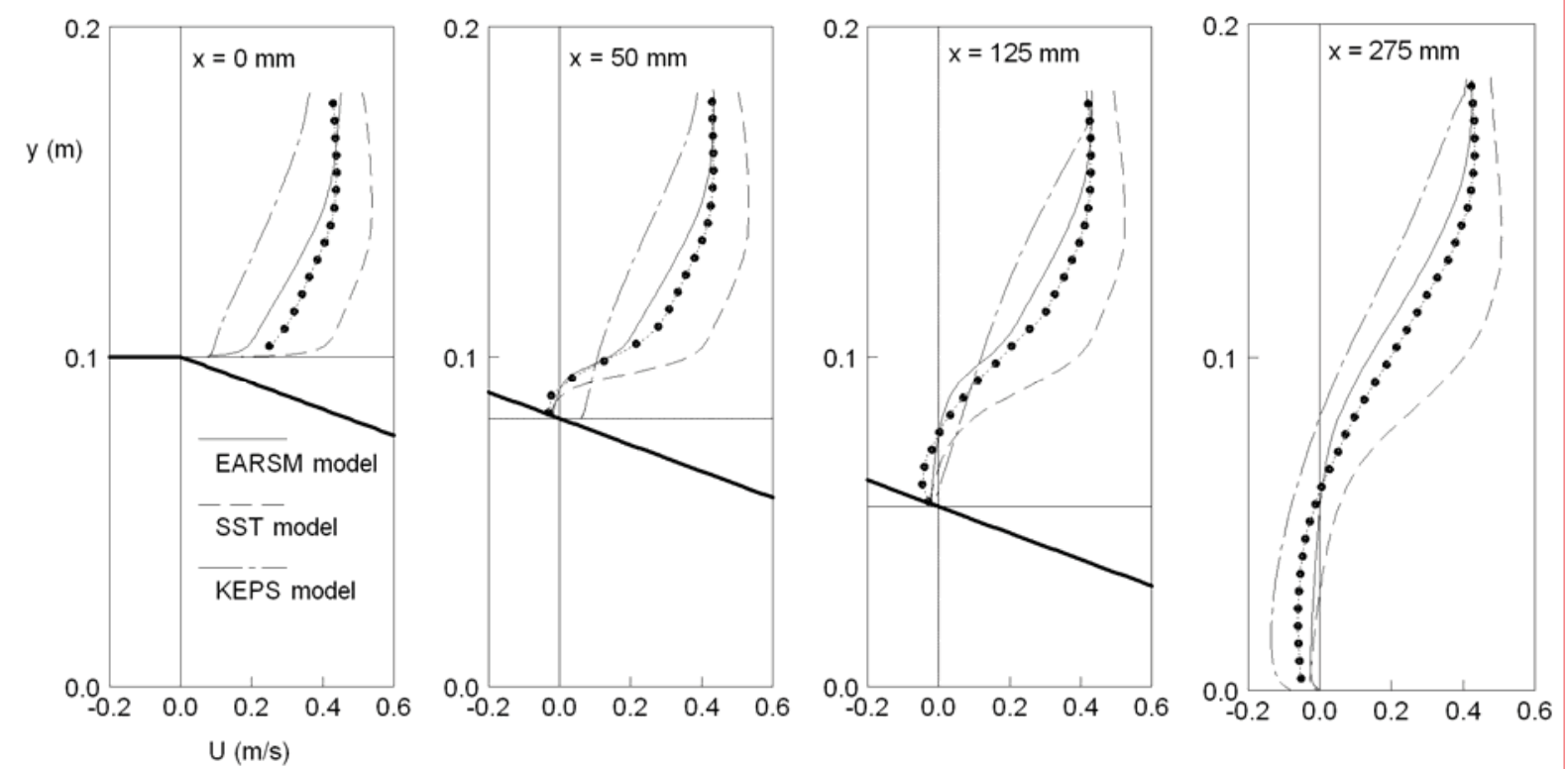

Fig. 3 Mean velocity profiles in the channel with the inclined step

(full line - EARSM model; dashed line - SST model, dash-and-dot line $-k-\varepsilon$ model)

\section{Results}

The analysis of experimental and numerical results was concentrated mainly on the development of flow separation behind the inclined step and on the corresponding changes of free surface. Further, the origin and development of secondary flow near side walls of the channel behind the inclined step was investigated.

Fig. 3 shows mean longitudinal velocity profiles in the symmetry plane over the step and in the outlet channel. Velocity profiles obtained by the EARSM, the SST model, and by the $k-\varepsilon$ model are compared with experimental data from PIV technique. It follows from the comparison that the turbulence models based on the assumption of the turbulent viscosity are not able to predict correctly the complicated flow pattern in the relatively 
narrow open channel with an inclined step. A satisfactory agreement with experimental data was obtained for the EARSM model only. Due to 3D character of flow with secondary effects near side walls, the velocity profiles obtained by the SST and the $k-\varepsilon$ model in the middle plane are not believable.

The form of free surface and the extent of the separated region in the middle plane $z=0$ are compared in Fig.4 with experimental data. The free surface is in the whole investigated area slightly rippled only. The predicted separated region corresponds to predicted velocity profiles. The extent of the region of the separated flow obtained by the EARSM model is in a good accordance with the experiment, while the SST model gives a smaller separation region. The $k-\varepsilon$ model gives an unrealistic separation bubble in the area of the step bottom. The length of the separation region behind the inclined step is approximately $x_{s} / H \approx 7.2$, while the length predicted by the EARSM model is $x_{s} / H \approx 7.6$.

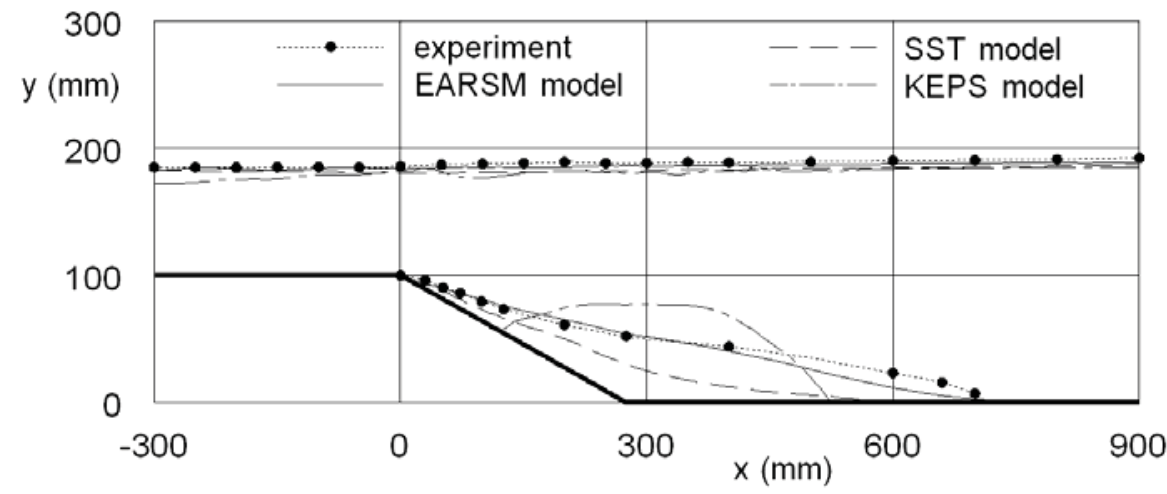

Fig.4 The extent of the separation region and the form of the free surface (full line - EARSM model; dashed line - SST model, dash-and-dot line $-k-\varepsilon$ model)

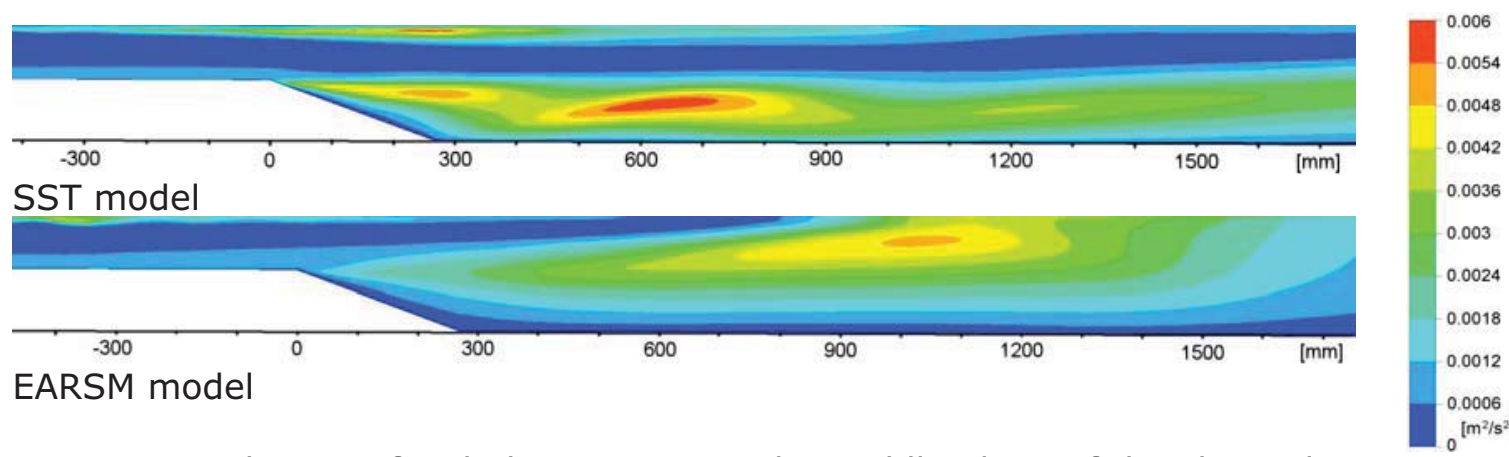

Fig.5 Distribution of turbulent energy in the middle plane of the channel

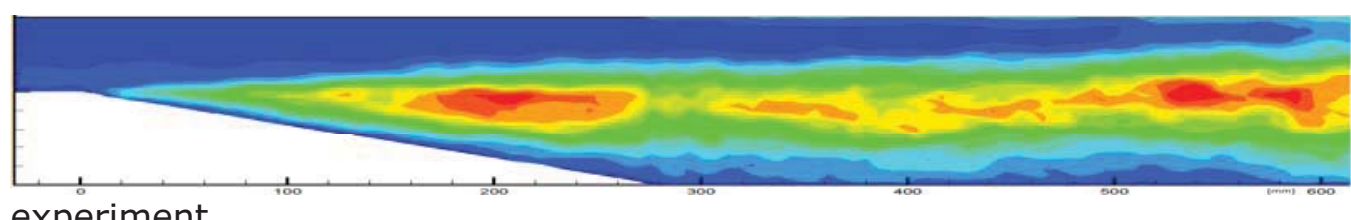

experiment

Fig. 6 Distribution of normal stress in the middle plane of the channel

The distribution of turbulent energy in the middle plane of the channel determined by means of the SST and the EARSM model is given in Fig. 5. According the SST model the 
maximum of turbulent energy is more noticeable in the mixing region immediately behind the flow reattachment. The maximal values of turbulent energy obtained by the EARSM model are smaller and the maximum moves to the proximity of free-level. The distribution of the longitudinal component of turbulent normal stress obtained by the PIV technique behind the step is given in Fig. 6 for comparison.

\section{Conclusions}

The investigation of the subcritical free-surface flow over a backward-facing inclined step in a rather narrow channel showed the complicated three-dimensional character of flow. The relatively long separation region behind the inclined step occurs in the subcritical regime while the free-level is slightly rippled only. Secondary flows arise behind the step edge near side walls partly close to the channel bottom and partly to the free-level.

It follows from the testing of turbulence models that models based on the turbulent viscosity are not able to predict correctly the complicated flow pattern in the studied case. A satisfactory agreement with experimental data was obtained for the EARSM model only. The SAS model gives very similar numerical results as the original SST model.

\section{ACKNOWLEDGEMENT}

The work was supported by the grant project No.103/09/0977 of the Czech Science Foundation and by the Research Plan AV0Z20760514.

\section{REFERENCES}

[1] Zubík P., Šulc J., Příhoda J., Sedlář M.: Experimental and numerical modelling of fluid flow with separation, In: Proc. $9^{\text {th }}$ Conference Power System Engineering, Thermodynamics and Fluid Flow, CD-ROM, 8p., Plzeň, 2010 (in Czech)

[2] Launder B.E., Sharma B.I.: Application of the energy-dissipation model of turbulence to the calculation of flow near spinning disc, Letters in Heat and Mass Transfer, 1, 131-138, 1974

[3] Menter F.R.: Two-equation eddy-viscosity turbulence models for engineering applications, AIAA J., 32, 1598-1605, 1994

[4] Menter F.R., Egorov Y. A Scale-Adaptive Simulation Model using Two-Equation Models, AIAA Paper 2005-1095, 2005

[5] Grotjans H., Menter F.R.: Wall functions for general application CFD codes, Proc. ECCOMAS 98, John Wiley \& Sons, 1112-1117, 1998

[6] Egorov Y., Menter F.R.: Development and application of SST-SAS turbulence model in the DESIDER project, Second Symposium on Hybrid RANS-LES Methods, Corfu, Greece, 2007

[7] Wallin S., Johansson A.: A complete explicit algebraic Reynolds stress model for incompressible and compressible flows, J. Fluid Mechanics, 403, 89-132, 2000

[8] Hellsten A.: New advanced $k-\omega$ turbulence model for high-lift aerodynamics, AIAA Paper 2004-1120, 2004 